\title{
ON DUAL RADICALS AND RING ELEMENTS
}

\author{
B. DE LA ROSA and R. WIEGANDT \\ (Received 19 June 1986; revised 14 September 1986) \\ Communicated by R. Lidl
}

\begin{abstract}
If a property $P$ of ring elements satisfies conditions (a)-(d), then the largest homomorphically closed class having no non-zero $P$-element is a dual radical in the sense of Andrunakievič, and every dual radical can be obtained in this way. Also properties defined by polynomials are considered and as an application we get various characterizations of the Behrens radical.
\end{abstract}

1980 Mathematics subject classification (Amer. Math. Soc.): 16 A 21.

Keywords and phrases: subdirectly irreducible ring, radical class, upper radical, special radical, dual radical, Behrens radical.

\section{Introduction}

The role of properties of elements in defining concrete radicals is well known. A systematic approach to the radical producing effect (as such) of element properties, may be found in [7]. The purpose of this paper is to continue this investigation of radicals as functions of element properties. We shall consider associative rings only.

Our interest is focused on a class of radicals which may be characterized as uniquely determined largest homomorphically closed classes of rings which are void of non-zero elements with some definite properties. These radicals are obtained by imposing two conditions on the element properties $P$ concerned, namely that $P$-elements be invariant under homomorphisms, and that they retain their $\boldsymbol{P}$-character relative to ideals which may contain them.

(C) 1988 Australian Mathematical Society $0263-6115 / 88 \$ A 2.00+0.00$ 
Among the radicals thus obtained we isolate a family of special radicals $\Re$ with the following representation:

$$
\left\{\begin{array}{l}
\Re \text { is the upper radical determined by a special class of } \\
\text { subdirectly irreducible rings, the hearts of which have non-zero } \\
P \text {-elements }
\end{array}\right.
$$

This is done by imposing two additional conditions on $P$, namely that $P$-elements of any ideal of a ring be $P$-elements of the ring as well, and that non-zero $P$-elements do not occur in trivial rings. Our two main results yield the fact that the representability of a radical $\Re$ in the form (*) is equivalent with the duality of $\Re$ in the sense of Andrunakievič.

We also emphasize the simple polynomial property

$$
\text { " } a \cdot f(a)=0 \text {, where } f(X) \text { is any polynomial in } \mathbb{Z}[X] "
$$

as a radical producing element property, and as an application we characterize the Behrens radical in a variety of ways.

\section{Radicals representable through special element properties}

Let $P$ be a property an element of a ring may possess. We shall consider the following conditions; none, some or all of which may be satisfied by the property $P$ concerned.

(a) If $a$ is a P-element of a ring $A$ and $\psi$ any homomorphism of $A$, then $\psi(a)$ is a $P$-element of $\psi(A)$.

(b) If $a$ is a $P$-element of $a$ ring $A$ and $a \in I \triangleleft A$, then $a$ is a $P$-element of the ring I.

(c) If $a \in I \triangleleft A$ is a P-element of the ring I then a is a P-element of the ring $A$.

(d) Trivial rings have no non-zero P-elements.

As examples we may mention idempotent element, (satisfying (a)-(d)); non-nilpotent element, (satisfying (b)-(d)); and unity element, (satisfying (a), (b) and (d)). Our interest in this paper centers on the following class of rings defined in terms of a property $P$ of elements:

$\mathfrak{B}_{P}=\{A:(\psi$ is a homomorphism of $A) \Rightarrow \psi(A)$ has no non-zero $P$-elements $\}$ The following statement is evident.

LEMMA 1. If $P$ is any property of elements then $\mathfrak{Q}_{P}$ is the uniquely determined largest homomorphically closed class of rings having no non-zero P-elements. 
For completeness we restate Theorem 2 of [7] as our

THEOREM 1. If $P$ is a property of elements which satisfies conditions (a) and (b) then $\mathfrak{\mathscr { B }}_{P}$ is a radical class.

Adding conditions (c) and (d) to the attributes of the property $P$ has a considerable influence on the resulting radical $\mathfrak{Q}_{P}$. This is exhibited in our next result.

THEOREM 2. Let $P$ be a property of elements which satisfies conditions (a)-(d). Then $\mathfrak{Q}_{P}$ is the upper radical $\mathscr{U} \mathfrak{M}$ of the special class $\mathfrak{M}$ of subdirectly irreducible rings the hearts of which have non-zero P-elements.

For the proof of this theorem we shall need the following simple consequence of Zorn's Lemma.

Lemma 2. If $a$ is a non-zero element of a ring $A$ then $A$ has a subdirectly irreducible homomorphic image $A / M$ with the non-zero element $a+M$ in its heart.

Proof of Theorem 2. If $A \notin \mathfrak{G}_{P}$ then $A$ has an image $B$ with a non-zero $P$-element. It follows by Lemma 2 and conditions (a) and (b) that $B$, and hence $A$, has a non-zero image in $\mathfrak{R}$, showing that $A \notin \mathscr{U} \mathfrak{R}$. Thus we have that $\mathscr{U} \mathfrak{M} \subset \mathfrak{Q}_{P}$. Conversely, if $A \notin \mathscr{U} \mathfrak{M}$ then $A$ has a non-zero image in $\mathfrak{M}$. Such an image of $A$ must (by (c)) have a non-zero $P$-element, and so $A \notin \mathfrak{S}_{p}$. Hence $\mathfrak{S}_{P} \subset \mathscr{U} \mathfrak{M}$; and we have $\mathfrak{Q}_{P}=\mathscr{U} \mathfrak{R}$.

It remains to show that $\mathfrak{M}$ is a special class of rings: Let $T$ be any ring in $\mathfrak{M}$. Then the heart $H(T)$ has a non-zero $P$-element. By (d) we have that $H(T)$ is a simple idempotent ring. It follows that $T$ is a prime ring. The hereditariness of $\mathfrak{M}$ is now also immediate. (See e.g. [4], Lemma 76.) Since every essential extension of a subdirectly irreducible ring with idempotent heart has the same heart, the class $\mathfrak{M}$ is also closed under essential extensions.

We shall refer to an element property $P$ which satisfies all four conditions (a)-(d) as a special element property, and in this case denote the upper radical $\mathfrak{S}_{P}$ of the special class of subdirectly irreducible rings the hearts of which contain non-zero $P$-elements, by the symbol $\mathfrak{S}^{P}$.

Next we consider element properties defined by polynomials. Let $f$ be a polynomial in one variable over the integers and define the property $P f$ as follows: an element $a \in A$ is a Pf-element of the ring $A$ if af $(a)=0$.

THEOREM 3. For any polynomial $f(X) \in \mathbb{Z}[X], \mathfrak{Q}_{P f}$ is a radical. Moreover, either $\mathfrak{Q}_{P f}$ does not contain all trivial rings, or Pf is a special element property, and hence $\mathfrak{\mathscr { Q }}_{P f}=\mathfrak{S}^{P f}$. 
Proof. Since $P f$ obviously satisfies conditions (a) and (b), $\mathfrak{\mathcal { Q }}_{P f}$ is a radical. Furthermore, it is clear that $P f$ satisfies (c). Finally, if $\mathfrak{G}_{P f}$ contains all trivial rings then condition (d) holds for $P f$.

Before formulating a converse to Theorem 2, we recall a construction by Andrunakievic, [1]: If $\Re$ is a supernilpotent radical then the upper radical $\Re_{\phi}$ of the class of subdirectly irreducible rings with $\Re$-semisimple hearts is a special radical, and $\Re \subset \Re_{\phi}$.

THEOREM 4. Let $\Re$ be a supernilpotent radical and define the property $P$ as follows:

(n) a non-zero element $a \in A$ is a P-element of the ring $A$ if $(a \notin I \triangleleft A) \Rightarrow$ $a+I \notin \Re(A / I)$.

(z) 0 is a $P$-element in each ring.

Then $P$ is a special element property, and $\mathfrak{S}^{P}=\Re_{\phi}$.

Proof. To prove the validity of (a), let $a \neq 0$ be a $P$-element of $A$ and $\psi$ a homomorphism of $A$. If $\psi(a) \neq 0$, let $K \triangleleft \psi(A)$ with $\psi(a) \notin K$. Then $a \notin \psi^{-1}(K)$ so that $a+\psi^{-1}(K) \notin \Re\left(A / \psi^{-1}(K)\right)$. Hence we obtain $\psi(a)+K \notin$ $\Re(\psi(A) / K)$.

Next let $a \neq 0$ be a $P$-element of $A$ and $K$ any ideal of $A$ with $a \in K$. Suppose that $a \notin M \triangleleft K$, and set $\Re(K / M)=L / M$. Assume that $a \in L$. Let $M^{*}$ denote the ideal of $A$ generated by $M$. By Andrunakievič's Lemma we have that

$$
\left(L / M^{* 3}\right) /\left(M / M^{* 3}\right) \simeq L / M \in \Re,
$$

and we know that $M / M^{* 3} \in \Re$ because $\Re$ contains the nilpotent rings. Since $\Re$ is closed under extensions we conclude that $L / M^{* 3} \in \mathfrak{R}$. Hence we have that

$$
a+M^{* 3} \in L / M^{* 3} \subset \Re\left(K / M^{* 3}\right) .
$$

Since $\Re\left(K / M^{* 3}\right)$ is an $\Re$-radical ideal of $A / M^{* 3}$ it follows that $a+M^{* 3} \in$ $\Re\left(A / M^{* 3}\right)$, a contradiction. Thus we have that $P$ satisfies (b).

To prove that (c) holds we consider a non-zero $P$-element $a$ of an ideal $I$ of a ring $A$. Let $K \triangleleft A$ such that $a \notin K$. Then $a \notin I \cap K \triangleleft I$, so that $a+I \cap K \notin$ $\Re(I / I \cap K)$. Hence we must have that $a+K \notin \Re((I+K) / K)=$ $((I+K) / K) \cap \Re(A / K)$. It follows that $a+K \notin \Re(A / K)$.

The validity of (d) is immediate by definition of $P$ and the fact that trivial rings are $\Re$-radical. Thus we have shown that $P$ is a special element property.

Finally, it easily follows that the $\Re$-semisimple hearts on the one hand and the hearts containing non-zero $P$-elements on the other, constitute exactly the same class of prime simple rings. Hence we have that $\Re^{P}=\Re_{\phi}$. 
By Theorems 2 and 4 we have

CoRollary 1. A radical $\Re$ is representable in the form $\mathfrak{S}^{P}$ if and only if $\mathfrak{R}$ is a dual radical, that is, $\Re=\Re_{\phi}$.

This means that certain radicals have no representations within our scheme. Theorem 2 yields the following sufficient condition for non-representability (see also Theorem 3).

COROLlaRY 2. Let $P$ be any special element property, and $f(X) \in \mathbf{Z}[X]$ any polynomial such that $\mathfrak{Q}_{P f}=\mathfrak{Q}^{\text {Pf }}$. If $\Re$ is a radical with $\beta \subset \Re$ but $\beta_{\phi} \not \subset \Re$, then $\mathfrak{R} \neq \mathfrak{\mathfrak { Q }}^{P}$ and $\mathfrak{\Re} \neq \mathfrak{\mathfrak { Q }}^{P f}$.

These non-representable radicals include well known (even special!) ones, like the prime radical $\beta$, the Levitzki radical $\mathscr{L}$, the nil radical $\mathscr{N}$ and the Jacobson radical $\mathscr{J}$ (see [4]).

Let us observe that non-representable supernilpotent radicals may have lower radical representations of the form $\mathfrak{S}_{P}$ where $P$ is non-special. For instance, $\mathscr{N}=\mathfrak{Q}_{T}$ where " $T$-element" means "non-nilpotent element"; a property which satisfies (b), (c) and (d) only. This may even happen in the case where the radical concerned is a dual radical. For instance defining " $U$-element" to mean "unity element" we see that this property satisfies (a), (b) and (d) only. By [6], Theorem 1 we have that $\mathfrak{Q}_{U}=\mathscr{G}$, the Brown-McCoy radical. We note here that $\mathscr{G}=\mathfrak{G}^{P}$, where " $P$-element of $A$ " means the unity element of a simple ideal of $A$ ".

\section{An example: the Behrens radical}

One of the most obvious candidates for a special element property is

$\mathrm{Pl}$ : the element $a \in A$ is idempotent.

In view of [5], Theorem 3 , the corresponding radical $\mathfrak{Q}^{\mathrm{P} 1}$ is the Behrens radical, that is, the upper radical of the class of subdirectly irreducible rings having non-zero idempotent elements in their hearts. (See [3] and [2].) We now consider the following list of element properties which will be proved to be suitable alternatives for $\mathrm{P} 1$.

P2: the element $a \in A$ is a cyclotomic element of $A$, that is, there exists a cyclotomic polynomial $\Phi_{n}(X)=\prod_{i=1}^{\varphi(n)}\left(X-\alpha_{i}\right)$ such that $a \Phi_{n}(a)=0$. (A priori the coefficients of $\Phi_{n}(X)$ are complex numbers, but we know that they are in fact rational integers.) 
P3: the element $a \in A$ satisfies $a^{n}-a=0$ for some $n \geqslant 3$.

P4: the element $a \in A$ satisfies $a^{n}+a^{n-1}+\cdots+a=0$ for some $n \geqslant 2$.

P5: the element $a \in A$ is von Neumann regular, i.e. there exists an element $x \in A$ such that axa $=a$.

It is clear that each of the properties $P j,(1 \leqslant j \leqslant 5)$, is special. Our next result shows that they all define the same radical, yielding various characterizations $\mathfrak{Q}^{P j}$ of the Behrens radical.

THEOREM 5. The following conditions on a ring $A$ are equivalent:

(1) A contains a non-zero idempotent element.

(2) A contains a non-zero cyclotomic element.

(3) A contains a non-zero element a such that $a^{n}-a=0$ for some $n \geqslant 3$.

(4) A contains a non-zero element a such that $a^{n}+a^{n-1}+\cdots+a=0$ for some $n \geqslant 2$.

(5) A contains a non-zero von Neumann regular element.

Proof. (1) $\Rightarrow(2)$ : If $a^{2}=a,(a \neq 0)$, then for the cyclotomic polynomial $\Phi_{2}(X)=X-1$ we have $a \Phi_{2}(a)=0 .(2) \Rightarrow(3)$ : Let $0 \neq a \in A$ such that $a \Phi_{n}(a)$ $=0, \Phi_{n}(X)=\prod_{i=1}^{\phi(n)}\left(X-\alpha_{i}\right)$. If $n=1$ we have $\Phi_{1}(X)=X-1$, so that $a^{2}-a$ $=0$ and hence $a^{3}-a=0$. If $n \geqslant 2$ there exists a $g(X) \in \mathbb{Z}[X]$ such that $\Phi_{n}(X) g(X)=X^{n}-1$. It follows that $a \Phi_{n}(a) g(a)=a^{n+1}-a=0$. (3) $\Rightarrow$ (4): Let $0 \neq a \in A$ such that $a^{n}-a=0$, with $n \geqslant 3$ minimal. Then $a^{n-1} \neq 0$, and $\left(a^{n-1}\right)^{2}=a^{2 n-2}=a^{n} \cdot a^{n-2}=a \cdot a^{n-2}=a^{n-1}$. Now the nonzero element $b=$ $-a^{n-1}$ satisfies $b^{2}+b=0$. (4) $\Rightarrow(1)$ : Let $0 \neq a \in A$ such that $a^{n}+a^{n-1}$ $+\cdots+a=0$ for some (minimal) $n \geqslant 2$. Then $a^{n} \neq 0$, and

$$
\begin{aligned}
\left(a^{n}\right)^{2} & =a^{n+1} \cdot a^{n-1}=\left[a^{n+1}+\left(a^{n}+\cdots+a\right)\right] a^{n-1} \\
& =\left[a\left(a^{n}+\cdots+a\right)+a\right] a^{n-1}=a^{n}
\end{aligned}
$$

Hence $a^{n}$ is a non-zero idempotent element.

Finally, (1) $\Rightarrow(5)$ is obvious; and $(5) \Rightarrow(1)$ follows from $($ axa $=a \neq 0) \Rightarrow$ $(a x)^{2}=a x \neq 0$.

\section{References}

[1] V. A. Andrunakievic, 'Radicals of associative rings I', Mat. Sb. 44 (1958), 179-212; Amer. Math. Soc. Transl. 52, 95-128.

[2] V. A. Andrunakievix, 'Radicals of associative rings II,' Mat. Sb. 55 (1961), 329-346; Amer. Math. Soc. Transl. 52, 129-149.

[3] E. A. Behrens, 'Nichtassoziative Ringe', Math. Ann. 127 (1954), 441-452. 
[4] N. J. Divinsky, Rings and radicals (University of Toronto Press, 1965.)

[5] R. E. Propes, 'A characterization of the Behrens radical,' Kyungpook Math. J. 10 (1970), 49-52.

[6] B. de la Rosa and R. Wiegandt, 'Characterizations of the Brown-McCoy radical,' Acta Math. Hungar. 46 (1985), 129-132.

[7] R. Wiegandt, 'Radicals of rings defined by means of elements,' Sitzungsber. Österreich. Akad. der Wiss., Math.-Natur. Kl. 184 (1975), 117-125.

Department of Mathematics

University of the

Orange Free State

Bloemfontein 9300

South Africa
Mathematical Institute of the Hungarian Academy of Sciences Budapest, V. Reáltanoda u. 13-15

Hungary 